\title{
Traffic Flow Sensing Using Wireless Signals
}

\author{
Xuting Duan, ${ }^{1, *}$ Hang Jiang1, Daxin Tian', Jianshan Zhou', Gang Zhou' ${ }^{1}$ and Wenjuan $E^{3,4}$, \\ Yafu Sun ${ }^{5}$, and Shudong Xia ${ }^{5}$ \\ ${ }^{1}$ Beijing Advanced Innovation Center for Big Data and Brain Computing, School of Transportation Science and \\ Engineering, Beihang University, Beijing 100191, China. \\ ${ }^{2}$ Research Institute of Highway, Ministry of Transport of the People's Republic of China, Beijing 100088, China. \\ ${ }^{3}$ School of Rail Transportation, Soochow University, Suzhou 215000, China. \\ ${ }^{4}$ Suzhou Automotive Research Institute, Tsinghua University, Suzhou 215000, China. \\ China TransInfo Technology Co., Ltd, Beijing 100085, China. \\ [e-mail: duanxuting@buaa.edu.cn] \\ ${ }^{*}$ Corresponding author: Xuting Duan
}

Received June 21, 2021; revised July 8, 2021; accepted July 14, 2021;

published October 31, 2021

\begin{abstract}
As an essential part of the urban transportation system, precise perception of the traffic flow parameters at the traffic signal intersection ensures traffic safety and fully improves the intersection's capacity. Traditional detection methods of road traffic flow parameter can be divided into the micro and the macro. The microscopic detection methods include geomagnetic induction coil technology, aerial detection technology based on the unmanned aerial vehicles (UAV) and camera video detection technology based on the fixed scene. The macroscopic detection methods include floating car data analysis technology. All the above methods have their advantages and disadvantages. Recently, indoor location methods based on wireless signals have attracted wide attention due to their applicability and low cost. This paper extends the wireless signal indoor location method to the outdoor intersection scene for traffic flow parameter estimation. In this paper, the detection scene is constructed at the intersection based on the received signal strength indication (RSSI) ranging technology extracted from the wireless signal. We extracted the RSSI data from the wireless signals sent to the road side unit (RSU) by the vehicle nodes, calibrated the RSSI ranging model, and finally obtained the traffic flow parameters of the intersection entrance road. We measured the average speed of traffic flow through multiple simulation experiments, the trajectory of traffic flow, and the spatiotemporal map at a single intersection inlet. Finally, we obtained the queue length of the inlet lane at the intersection. The simulation results of the experiment show that the RSSI ranging positioning method based on wireless signals can accurately estimate the traffic flow parameters at the intersection, which also provides a foundation for accurately estimating the traffic flow state in the future era of the Internet of Vehicles.
\end{abstract}

Keywords: Internet of Vehicles, RSSI, Traffic Flow Sensing, Cooperative Vehicle Infrastructure System. 


\section{Introduction}

The intelligent transportation system (ITS) is currently the frontier research field of world transportation. Cooperative vehicle infrastructure system (CVIS) is an essential part of the intelligent transportation system. CVIS mainly uses wireless communication and sensor detection to realize intelligent coordination and cooperation between vehicles to vehicles(V2V) and vehicles to infrastructures (V2I), fully utilize the road network resources, improve road network traffic safety and efficiency, ease traffic congestion, and optimize the overall road network goals [1, 2]. High-precision positioning technology is one of the critical technologies of CVIS [3, 4]. It is an essential guarantee for vehicles' safe driving and the key to realizing mutual perception between people and vehicles. The intelligent intersection is an important research direction of CVIS. The intersection's traffic flow state can be effectively introduced by collecting the passing vehicles' positioning data. It facilitates intelligent regulation of the intersection signal lights. Also, collecting the vehicle's positioning data is beneficial to the autonomous vehicle's formation, which improves the traffic efficiency of the future intersection [5]. A coordinated signal control system for urban ring roads based on a vehicleinfrastructure interconnection environment is developed [6-8] to improve urban ring roads' operational efficiency and reduce traffic delays. The system can adjust the signal timing parameters such as cycle length, green segmentation, offset, etc., improving road network efficiency. The main road's signalized intersection is divided into several subgroups, and the signal is optimized within each subset [9, 10]. The model used a mixed-integer linear programming technique to ensure an optimal global solution and got the ideal trunk green wave belt. In [11], the authors propose a network aided traffic steering technique in 5G mobile network architecture. The 5G mobile systems can monitor network conditions and learn with network data with the machine learning algorithms. In [12], the authors use the EBF sketches method to combine Bloom Filter with an exponential histogram to query streams in the sliding window to identify heavy vehicles in the speed traffic streams. In [13], the authors propose an analysis method based on the priority of the wireless sensor networks (WSNs) in Highway Traffic to get the accurate location of the node. This approach also works well for V2X network environments.

The positioning problem can be divided into indoor positioning and outdoor positioning. At present, there have been a lot of researches on indoor positioning. Most indoor positioning systems use the RSSI based on the Wi-Fi signal [14-16]. There are usually two processing methods. According to the propagation loss model, one is to obtain the distance between the receiver and the transmitter by measuring the signal strength received at the receiver. Another way is to use the fingerprint information of the RSSI of Wi-Fi to establish the mapping relationship between the RSSI and the location in the current scene, and the positioning system is trained through the offline data [17].

Both of the methods have limitations. The limitation of the positioning method based on the signal propagation loss model is that the loss model cannot accurately describe the wireless signal's attenuation in various complex scenarios. And because of the multipath interference in complex scenes, it is difficult to model the signal attenuation accurately. On the other hand, the Wi-Fi receiving signal strength fingerprint positioning method's limitation is that the previous data collection and offline training cost is relatively high. And the scheme is only applicable to the static environment. After the environment changes significantly, the original RSSI location database needs to be updated $[18,19]$.

Recently, a lot of research on positioning focused on the channel state information (CSI) of Wi-Fi. CSI is different from RSSI in that it is a channel response from the physical layer. 
It describes the amplitude and phase of each subcarrier in the frequency domain [20, 21]. We can get multiple carrier responses relative to RSSI. More importantly, CSI is not susceptible to multipath effects, making it possible to achieve precise positioning using CSI. A devicefree state detection and localization algorithm based on Wi-Fi CSI and support vector machine (SVM) are proposed [22]. An indoor fingerprint recognition system based on CSI based on deep learning is proposed. DeepFi can effectively reduce the positioning error [23]. In [24], the authors convert the CSI measurements of multiple channels into a radio image, extract color and texture features from the radio image, learn the optimized depth features from the image features using a deep learning network, and estimate the position of a person using machine learning methods. In [25], the authors use the CSI information to achieve smoke detection. A channel state information component reconstruction (CSI-CR) algorithm is proposed to estimate the angle of arrival (AoA) of the human body reflected signals in a through-wall (TTW) scenario [26]. In [27, 28], the detection of indoor human activities is achieved using wireless signals. In [29, 30], CSI is used for achieving accurate indoor positioning with an accuracy of decimeters or even centimetres. In [31], the authors use the Doppler spread in the envelope of the received signal to estimate the mobile user's speed. CSI technology can also be used for network security protection on the Internet of Vehicles. The authors propose a reinforcement learning algorithm to identify rogue nodes using the channel state information of the communication link between vehicle-to-vehicle and vehicle-toinfrastructure to figure out a rogue attack problem [32]. In [33-35], the authors estimate unreliable channel information for capture-aware identification of mobile RFID tags, for the sake of traffic flow sensing in the future.

Compared with indoor positioning, the typical traffic scene is an environment that is relatively open outdoors. The outdoor multipath effect is relatively small, but the spatial range is relatively wide. The signal is mainly affected by the increase of the distance and the attenuation during the propagation process. At present, most of the research is focused on indoor positioning, and outdoor positioning technology mainly relies on global satellite positioning technology and base station positioning technology [36]. The former uses at least four space satellites at the same time to accurately locate objects. The latter is generally applied to mobile phone users. The mobile phone base station location service is also called locationbased service (LBS), which acquires the location information of the mobile terminal user through the network of the mobile telecommunication operator [37]. Our main research scenario is a typical urban cross signal control intersection. The scene radius is within 300 meters, and the Wi-Fi AP access point is set on the traffic signal. The vehicle on the lane is located by collecting the received signal strength RSSI value of the incoming and outgoing vehicles, thereby deriving the traffic flow's status information.

This paper proposes a sensing method for traffic flow state information of WLANs' crosssignal intersections based on the RSSI signal attenuation model. And a simulation experiment was carried out to verify.

In summary, the main contributions of this paper are as follows:

Firstly, we designed a positioning scheme based on the RSSI-ranging model applied to a typical urban cross signal intersection scene. The Wi-Fi signal strength value RSSI received by the vehicle node is measured. The RSSI ranging model is used to obtain the distance $\mathrm{d}$ between the vehicle and the traffic light, which is applied to derive the traffic flow parameters.

Secondly, we built a simulation platform for the Internet of Vehicles through sumo, $\mathrm{OMNeT}++$, and veins. First, we built a simulation scenario of a typical cross signal intersection. Then we built a car-network communication environment at the signalized intersection and collected the signal strength indicator RSSI received by the vehicle node to 
locate the vehicle. Finally, we derive the traffic flow parameters based on the vehicle's positioning data.

Finally, we show the experimental results of the simulation experiment and analyze the results. We summarized the shortcomings of this work and looked forward to future work.

\section{Related work}

Traffic flow detection plays an important role in ITS. Real-time road traffic parameter collection and accurate road congestion evaluation are the prerequisites for applying better traffic congestion avoidance strategies to improve the traffic flow [39,40]. The traditional traffic flow detection methods mainly include ultrasonic detection, induction coil detection and geomagnetic detection. The magneto-electric induction detecting method, in which loop detectors acquire the section flow data or geomagnetic detectors placed underground at a certain road section [41,42]. The floating vehicle method extracts traffic parameters from the trajectory data by intelligent onboard devices, such as bus floating data and taxi floating data [43]. For example, the video image detecting methods include the traffic parameter collection by electronic-police camera or the UAV [44] and the radar detecting methods. The floating vehicle method can be applied to micro and macro traffic flow estimation. The magnetoelectric induction detecting method can only be applied to microscopic traffic flow estimation. The video image detecting methods can also be divided into traditional visual detection methods and deep learning-based methods. For traditional visual methods, in [45], the authors combine histogram of oriented gradient (HOG) extracting features with SVM training detection model as the vehicle detection method. In [46], Gaussian mixture model (GMM) is employed to model the dynamic background of the traffic scene. Then, the binary foreground contours are extracted by image subtraction. The real and complete vehicles are obtained to detect and monitor the binary vehicle contours' location and the current frame. In [47], the authors proposed a pyramid-YOLO network for detecting vehicles in dense scenes, which can effectively detect small-size and occluded vehicles. And based on the detection and counting results, an estimation model is proposed to estimate traffic flow parameters of volume, speed and density. In [48], the authors collect the vehicle parameters by the radar data whose features are analyzed from the dimensions of single parameter sampling characteristics and multiparameter relationships. Further, the correlations of different traffic flow parameters are given using the grey correlation analysis method.

This paper proposes a traffic flow parameter estimation method based on wireless signal RSSI data to extend indoor positioning technology to outdoor intersection scenes. This provides a practical basis for traffic flow estimation in the IoV environment.

In conclusion, the method based on deep learning vision is more expandable and accurate to estimate traffic flow compared with traditional visual methods. However, this method relies on data set support and is also influenced by environmental factors such as illumination. The estimation method of traffic flow parameter based on the millimetre-wave radar is less affected by environmental factors. The device is easy to deploy and maneuverable. The estimation method of traffic flow parameter based on wireless signal RSSI data is suitable for the network environment of the Internet of Vehicles under multi-scale conditions. This can provide a basis for traffic flow estimation in the IoV environment. 


\section{System Architecture and Problem Formulation}

Our main work is shown in Fig. 1. First of all, we use the SUMO, OMNeT++, and Veins to build a network simulation platform, which realizes the construction of the single point of intersection and the configuration of the network environment. Then we collect the vehicle original RSSI trajectory data required by the simulation experiment and process it with Gaussian filter. Then we calibrate the parameters of the ranging model. Finally, we collect RSSI time track data, set the simulation step size, and then obtain the vehicle's space-time track map to calculate the traffic flow parameters.

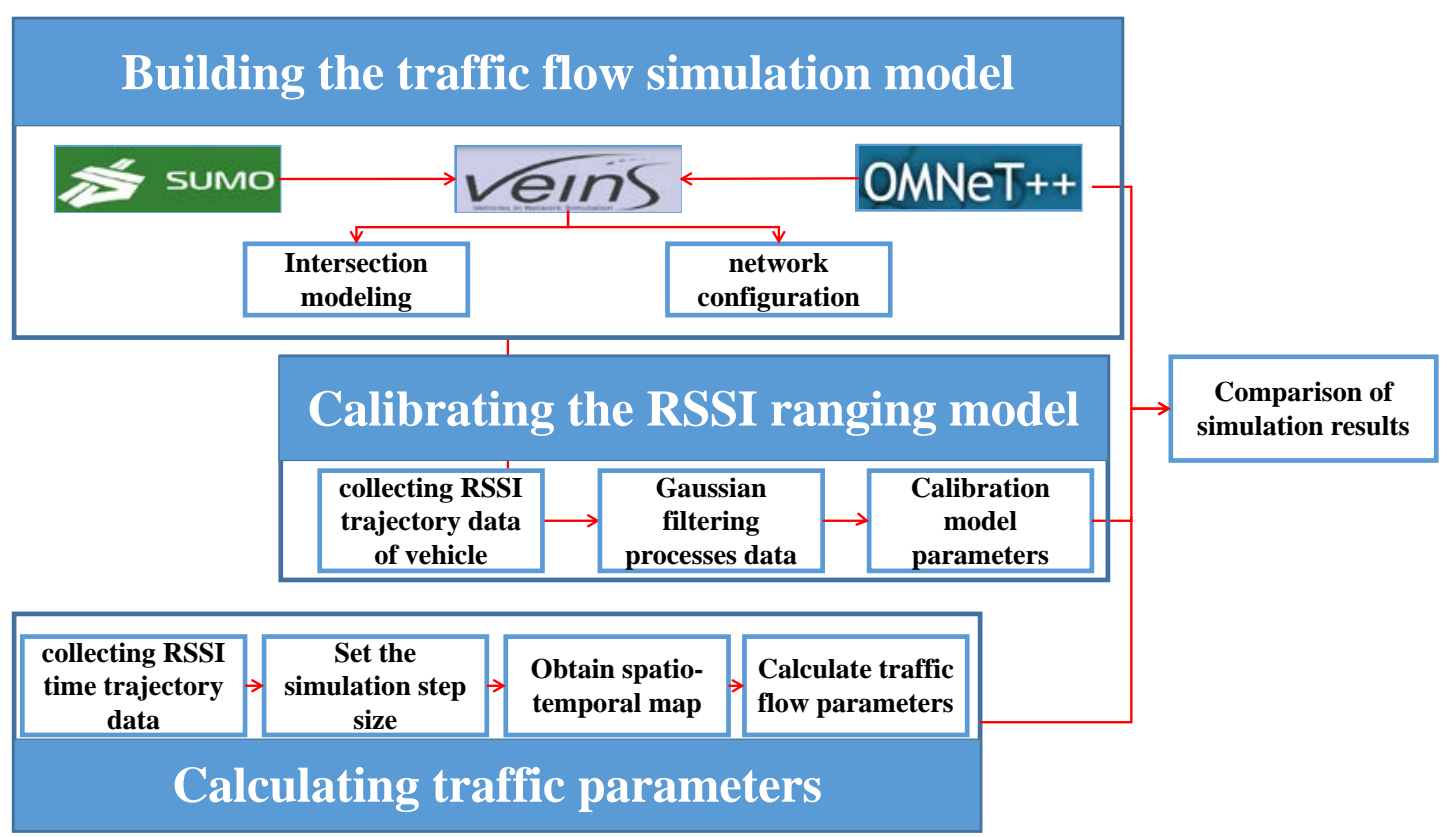

Fig. 1. System flowchart.

\subsection{THE RSSI RANGING MODEL}

In an actual outdoor environment, the intersection of the cross signal is not as small as the indoor space. The radio waves do not have too much refraction and reflection during the transmission process. Instead, they are on the sides of the road, tall buildings, or apartments. And the multipath effect is caused by ground reflections, but there is not much shadow obscuration in the lane. Besides, it should be accompanied by large-scale and medium-scale propagation fading of electric waves. We choose the logarithmic distance attenuation model[16] to characterize the Wi-Fi signal's attenuation at the intersection.

$$
\operatorname{RSSI}(d)=\operatorname{RSSI}\left(d_{0}\right)+10 \log \frac{d}{d_{0}}+X_{0}
$$

Where $d$ is the interval between the wireless signal receiving terminal and the transmitting terminal, is expressed as the distance of the reference point, generally selected as $1 \mathrm{~m}$. $\mathrm{n}$ is a fixed value coefficient determined by the surrounding environment, indicating the degree to which the radio wave's attenuation increases with the distance $\mathrm{d}$, and $\mathrm{n}$ is the signal attenuation factor. $X_{0}$ is a Gaussian random distribution whose variance is $\sigma$, where $\sigma$ is 3 to $14 \mathrm{dBm}$.

We can get a ranging model based on RSSI signal attenuation.

$$
\mathrm{d}=10^{\frac{A_{0}-R S S I}{10 n}}
$$


Therefore, according to the RSSI ranging model, we can calibrate the RSSI ranging model parameters by collecting the corresponding RSSI value and the distance d between the corresponding RSU and the vehicle receiving node in the simulation experiment. The first calibrated parameter is $\mathrm{K}$ : environmental attenuation factor, and $A_{0}$ is RSSI value when the reference distance is 1 meter.

\subsection{BUILDING A SIMULATION PLATFORM}

\subsubsection{Simulation experiment research scene}

The research scene is mainly the cross signal intersection. The experimental study scenario is shown in Fig. 2. We deploy the roadside unit RSU on the traffic signal at the cross-signal intersection, and the roadside unit RSU emits radio waves outward at the centre frequency of $5.89 \mathrm{GHz}$. The vehicle node enters the cross-signal intersection and receives the wireless signal from the roadside unit RSU. We collect the RSSI data received by each vehicle node per second. We can obtain the RSSI trajectory data of the cross-signal intersection's entrance lane to obtain the microscopic traffic parameters of the intersection (traffic flow rate, speed, and density). The research scene is a typical single-point crossroad intersection scene. This type of scene is mainly the most common single-point intersection scene in the city. Such scenes are prevalent in small cities and large cities, so they have the research's significance.

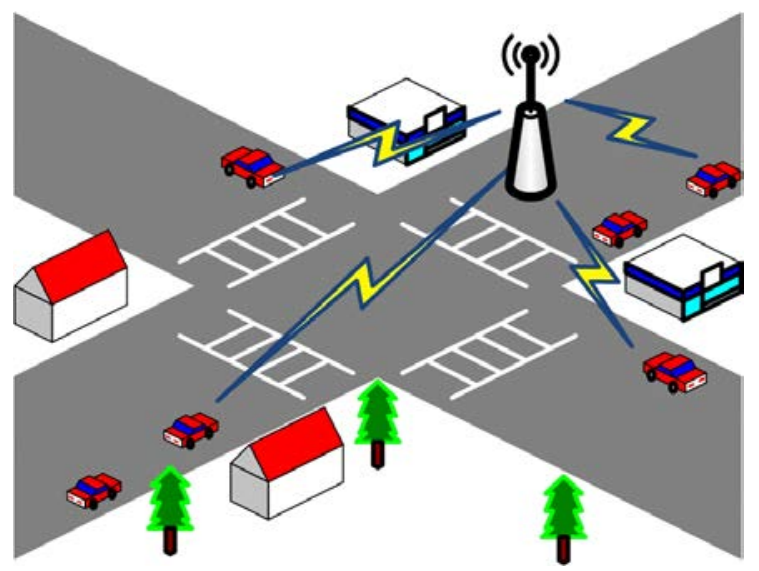

Fig. 2. Simulation scene.

\subsubsection{Establish a signal intersection model}

In [38], the authors propose a model for simulating real-world transportation networks by representing the irregular road networks with static and dynamic attributes. We use the above method to construct the simulation model of regular cross-shaped intersections. First, we mainly establish and use a single-point cross signal intersection model and then build a complex double cross signal intersection model. For common intersections, the distribution of traffic flow is different at simple intersections. The traffic flow distribution of common intersections is shown in Table $\mathbf{1}$. 
Table 1. The traffic flow distribution

\begin{tabular}{|c|c|c|c|c|}
\hline $\begin{array}{c}\text { Approaching } \\
\text { road }\end{array}$ & $\begin{array}{c}\text { Turn left } \\
\text { (veh/h) }\end{array}$ & $\begin{array}{c}\text { Straight } \\
\text { (veh/h) }\end{array}$ & $\begin{array}{c}\text { Turn right } \\
\text { (veh/h) }\end{array}$ & $\begin{array}{c}\text { Sum } \\
\text { (veh/h) }\end{array}$ \\
\hline West & 200 & 900 & 300 & 1400 \\
\hline East & 200 & 600 & 200 & 1000 \\
\hline South & 200 & 150 & 150 & 500 \\
\hline North & 200 & 150 & 150 & 500 \\
\hline
\end{tabular}

(1) Single-point cross signal intersection traffic signal timing setting

From the above traffic flow distribution table, the optimum signal period $\mathrm{C}$ can be calculated. The timing scheme is designed as follows.

We first set the yellow light time A to 3 seconds, the start loss to 3 seconds, and the full red time to 2 seconds.

Since the four import lanes have left more than 200 vehicles per hour, they are all set to turn left. Therefore, the phase of the signal light is controlled by four phases. Simultaneously, the saturated flow rate $\mathrm{S}$ of each lane is set to 1400 vehicles per hour.

The key flow rate ratios of the four phases calculated by (3) are $0.237,0.118,0.178,0.118$. Therefore, the total critical flow rate ratio is $\mathrm{Y}=0.65$ and less than 0.9 , indicating that the setting is reasonable.

$$
y_{i}=\frac{Q_{i}}{S}
$$

Where $y_{i}$ represents the critical flow rate ratio of the ith phase, $Q_{i}$ represents the phase critical flow, and $S$ represents the saturated flow rate of the lane.

And then, we can calculate the total loss duration $\mathrm{L}=20$ seconds by (4)

$$
\mathrm{L}=\sum_{k} l+I-A
$$

Where $\mathrm{L}$ is the total loss duration, $\mathrm{l}$ is the start loss, $\mathrm{I}$ is the green light interval, and $\mathrm{A}$ is the yellow light duration.

Then we calculate the optimal period is $C=100$ seconds by (5)

$$
\mathrm{C}=\frac{1.5 L+5}{1-Y}
$$

The total effective green time $G_{E}=80$ seconds is calculated by (6). The effective green time for each phase calculated by (7) is $35 \mathrm{~s}, 15 \mathrm{~s}, 15 \mathrm{~s}, 15 \mathrm{~s}$.

$$
G_{E}=C-L
$$

Where $G_{E}$ is the total effective green time.

$$
g_{i e}=G_{E} \cdot \frac{y_{i}}{Y}
$$

Where $g_{i e}$ is the effective green time of the ith phase.

The actual display green time of each phase is calculated by (8), and the start loss is exactly equal to the yellow light duration, so the effective green time is equal to the actual display green time.

$$
g_{i}=g_{i e}+l_{i}-A
$$

Where $g_{i}$ is the actual green light display time of the ith phase.

\section{(2) Single point cross signal intersection environment configuration}

In the actual cross signal intersection scene, there are vehicles in the lane and buildings on both sides of the lane, which causes multipath effects such as radio waves to occur during transmission. Therefore, when the sumo simulation software models the common intersection scene, it is necessary to establish the intersection model and establish the architectural model 
around the intersection. In the sumo software, the polygon.xml file is used to edit the required building type, shape, size, and position. The building model established by the experiment has 52 symmetrical forms. The actual single point cross signal intersection is as shown in Fig. 3

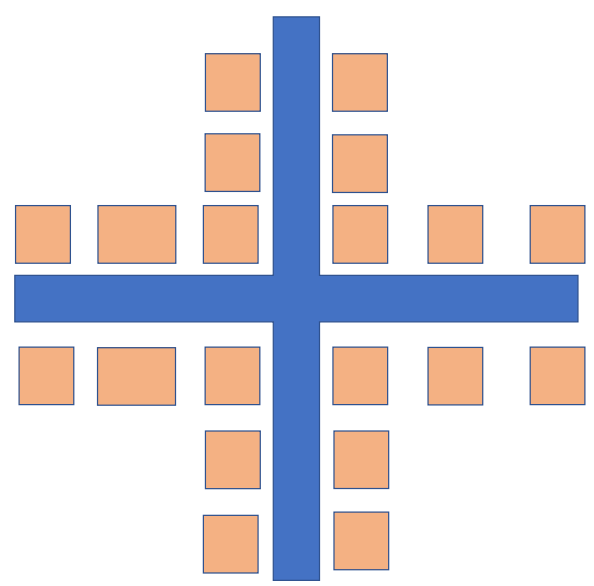

Fig. 3. Signal intersection model.

\subsection{BUILDING A SIMULATION PLATFORM}

We mainly realize real-time communication of network nodes through the following several modules. The Annotation Manager module mainly implements simulation visualization. The obstacle control module is mainly used to realize signal attenuation caused by radio waves encountering obstacles during transmission. The shadow barrier control module in Veins is provided by the environment's model around the intersection of the cross signal established by sumo. The Base World Utility module provides the simulation scenario's size for network simulation in the veins simulation. The Connection Manager module can determine the range of communication and the interference range of nodes in the wireless network according to the power $P_{t}$ of the signal transmitting terminal, the wavelength $\lambda$ of the wireless signal, the path loss coefficient $\mathrm{n}$ in the signal propagation loss model, and the minimum received power $P_{(r)}$ of the signal receiving terminal. The RSU module mainly realizes setting the roadside unit RSU in the intersection model and establishing a network communication model. The specific design and composition of the RSU module are shown in Fig. 4.

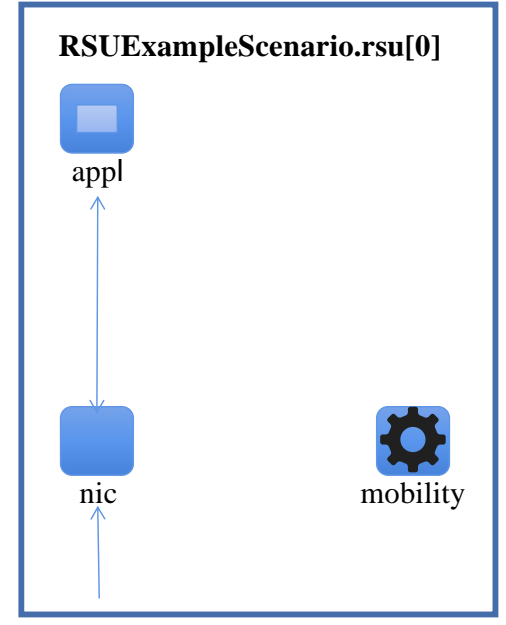

Fig. 4. RSU model. 
The RSU comprises three modules: appl (RSU network application layer), nic, and mobility. The nic module uses the nic module based on the IEEE802.11P protocol in the vehicle network architecture, as shown in Fig. 5. The nic module's physical layer adopts the phy802.11p communication module, while the MAC layer uses the communication module based on IEEE1609.4.The Phy802.11p module uses a bidirectional connection, first connected to the antenna module to obtain messages sent from the antenna. This is followed by an opposite connection to the MAC1609.4 module. The MAC layer is also responsible for communicating with the appl (network application layer) as the nic module's upper module. The mobility module is independent of the other two modules in the RSU module. The mobility module mainly sets the starting position of the RSU and mobility characteristics. In this article, the location of the RSU is set at the east entrance of the intersection.

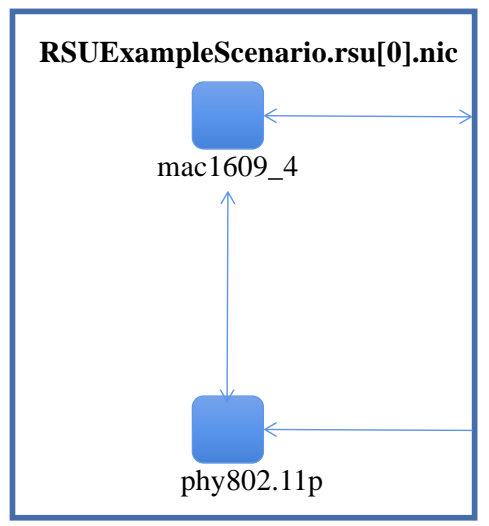

Fig. 5. V2X wireless communication model.

The vehicle nodes are constantly moving, so the Internet of Vehicles' network topology is constantly changing. The wireless communication methods between nodes are as follows: First, the application layer of the RSU node generates Beacon type data after initialization activation and then periodically sends a message to itself (RSU). The application layer processes any messages sent to itself to achieve the purpose of timed loops. The application layer then transmits the beacon message to the MAC layer. After receiving the beacon message, the MAC layer encapsulates it into a MAC frame. It then uses the CSMA/CA mechanism for channel contention to obtain a nextmacevent, the MAC layer converts the transceiver mode of the PHY layer. The physical layer then sends a control message to the MAC layer, and then the MAC layer sends the beacon message to the physical layer. After receiving the beacon frame sent by the MAC layer, the PHY layer encapsulates it into a data frame (Airframe) of the PHY layer and then sends the frame to the channel; then broadcasts the Airframe data frame to the antenna. After receiving the Airframe sent by the RSU, the basic physical layer in the vehicle node module processes the frame through the handle airframe function. The handle airframe function mainly uses three states for message processing. The first state mainly uses the filter signal function to obtain the signal's attenuation value by the logarithmic distance attenuation model. The second state then obtains the received signal power through the process signal function and determines whether to accept the signal data pack. The last third state is to use sendup to transfer the data up to the MAC layer. The mac layer in the vehicle node module receives the data sent by the PHY layer. It then sends it up to the application layer after unpacking, thereby completing the communication process between the RSU node and the mobile vehicle node. The vehicle node RSSI is acquired in the physical layer. 


\section{SIMULATION RESULTS AND DISCUSSION}

\subsection{Calibration measuring distance model}

We choose the log path shadow attenuation model as the ranging model. The carrier's centre frequency needs to be set to $5.89 \mathrm{Ghz}$, and the environmental impact factor $\mathrm{n}$ is set to 3.0. In the sumo simulation, we used a car to calibrate the west entrance parameters through the intersection.

\subsubsection{Preprocess raw data}

First, multiple original RSSI data at different distances are collected in the simulation, and then the original data is preprocessed. Multiple measurements of the RSSI at the same location will have Gaussian random interference. The Gaussian filter is used to select the RSSI value of the high probability range, and then the average is used to reduce the Gaussian random interference.

\subsubsection{Calibration model parameters}

The position $d$ of the vehicle node collected in the simulation process and the corresponding RSSI data are plotted by MATLAB as shown in Fig. 6. This shows that the relationship between distance $d$ and RSSI is logarithmic attenuation, which is in line with experimental expectations.

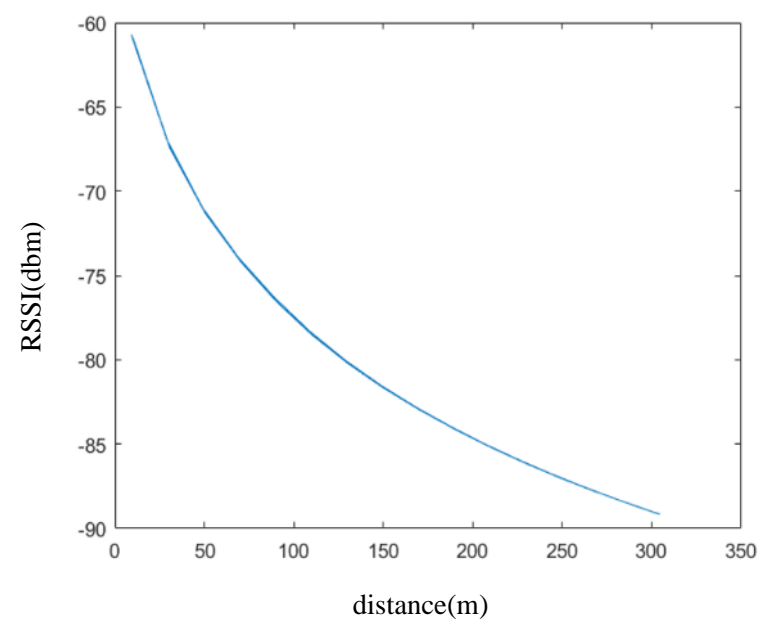

Fig. 6. Relationship between distance d and RSSI.

We can get (9) from the (2) so that lgd is x, the actual measured RSSI is y, and $A_{0}$ is the RSSI when the distance between RSU and the mobile node of the vehicle is $1 \mathrm{~m}$. So the fit of $A_{0}$ and n can be obtained by linear fitting through MATLAB. The fitting curve is as shown in Fig. 7.

$$
\mathrm{RSSI}=A_{0}-10 n l g d
$$




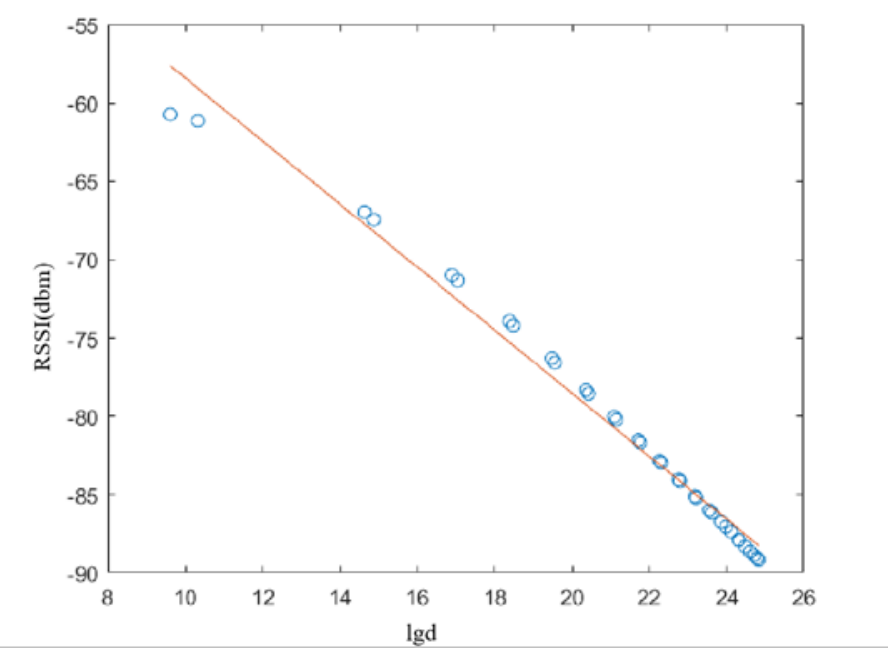

Fig. 7. The fitting curve of $\mathbf{A}_{\mathbf{0}}$ and $n$.

\subsection{Detecting traffic flow parameters of straight traffic flow at the west entrance of the actual single-point signal intersection}

The experimental scene is mainly that the phase of the signal light at the intersection is in the first phase, and the traffic in the east-west direction is straight through, the traffic volume in the west import is 900 hours per hour, and the east entrance is 600 vehicles per hour. And the vehicle travels at a constant speed. The terminal collects the Wi-Fi signal strength received by the west-imported vehicle, obtains the vehicle's distance by the ranging model, and draws the space-time trajectory image of each vehicle in the measurement time through the MATLAB, as shown in Fig. 8. We use MATLAB to linearly fit it to obtain the slope value of the curve. Each curve's average slope was averaged to obtain a vehicle traffic flow at a constant speed of $13.13 \mathrm{~m} / \mathrm{s}$. And the relative error was $5 \%$ compared with the actual $12.5 \mathrm{~m} / \mathrm{s}$. The average headway distance is $4.16 \mathrm{~s}$. Then, from the front of the car, the westbound direct traffic flow $\mathrm{Q}$ is 865 vehicles per hour, and the relative error is 3.8\%. Then, the relationship between the flow, velocity, and density of the traffic flow is available. The density of the straight westbound traffic is 66 vehicles per kilometre.

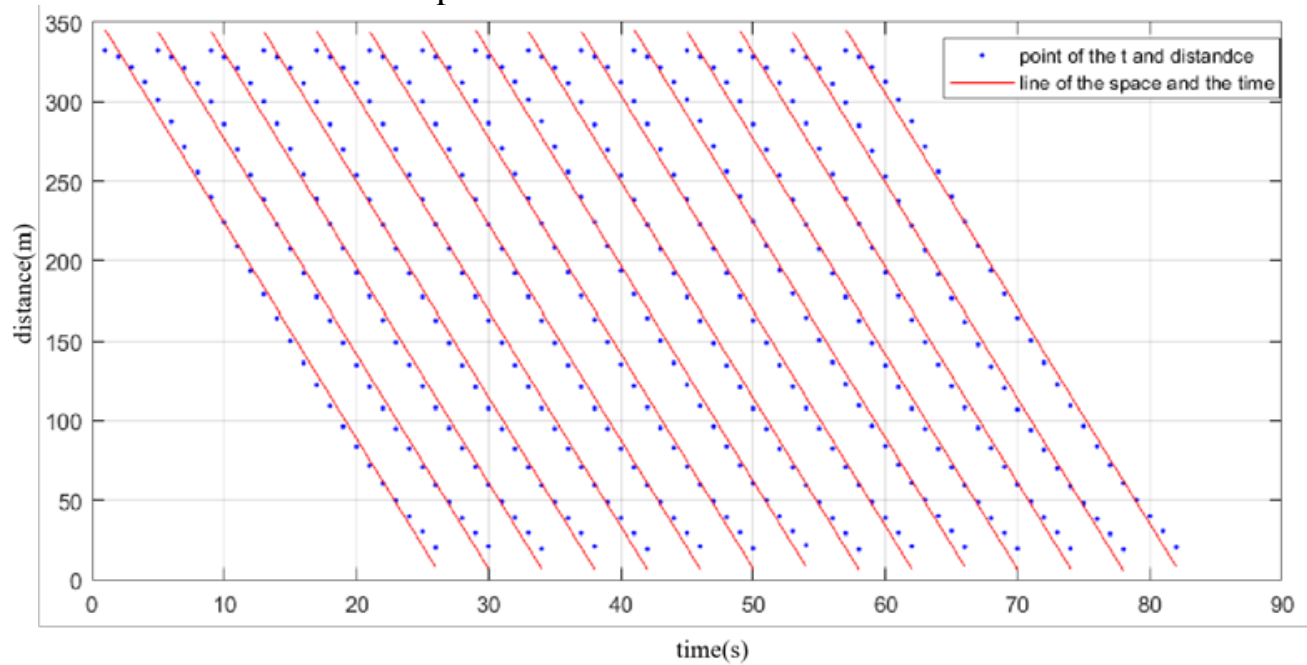

Fig. 8. The space-time trajectory of each vehicle. 


\subsection{Detect the queue length of left-turn traffic}

Dedicated left-turn lanes appear in the queue. The west-imported left-turn traffic volume is 200 vehicles per hour, and the traffic flow is also at a constant speed. During the test's redlight time, collect the vehicle's RSSI value entering the special left-turn lane of the west entrance and then obtain the vehicle's corresponding distance. The time and space diagram of each car drawn by MATLAB is shown in Fig. 9. When the red light is cut off, the distance between the first car and the distance signal light is 13.5 meters. The last car distance signal is 42.6 meters, so the distance between the first and the last one is 29.1 meters, and the distance between the vehicles when the queue is 5 meters, so you can get a queue length of 6 cars.

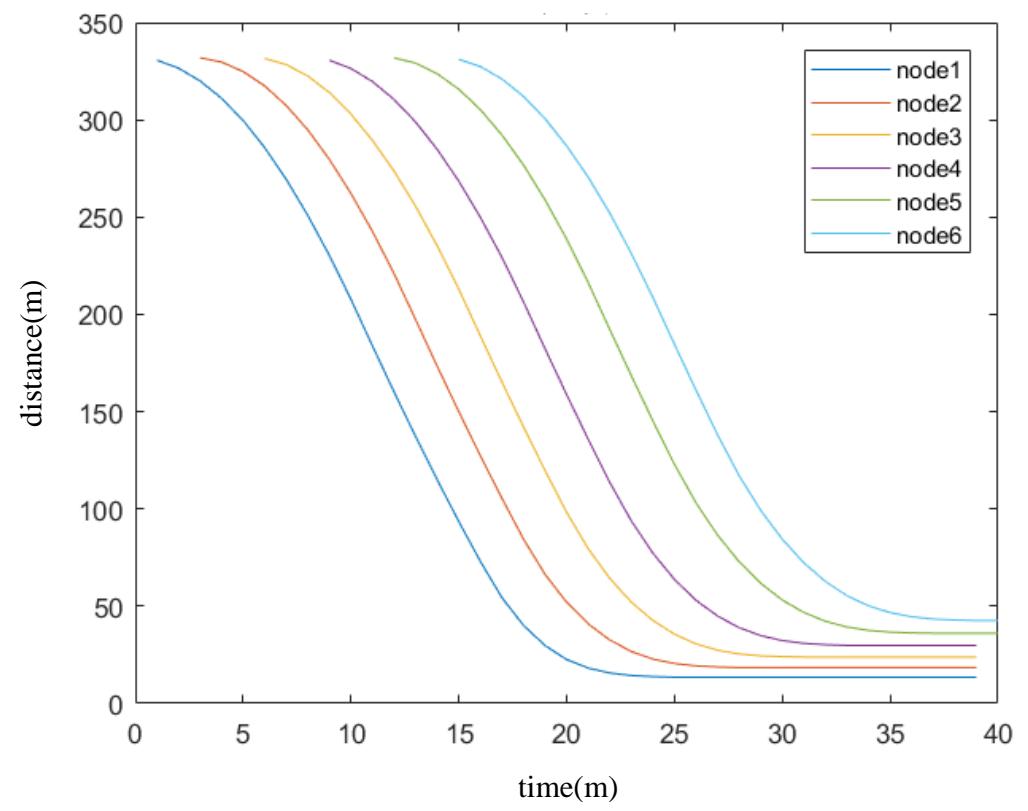

Fig. 9. The time and space diagram of each car.

\subsection{Discussion of the results}

First, we calibrate the RSSI based ranging model. The model is transformed from the logarithmic decay model. As shown in Fig. 6, we obtained the distance relationship between vehicle wireless signal RSSI data and RSU through measurement. The relationship is logarithmic decay. By fitting the logarithmic decay function, we obtained the parameters of the RSSI ranging model $A_{0}=-38.3361 \mathrm{dBm}, n=2.8876$. This fits the assumptions of the simulation scenario. Then we set the average speed of the traffic flow as $\mathrm{v}=12.5 \mathrm{~m} / \mathrm{s}$. Therefore, we measured the spatiotemporal trajectory diagram of the inlet channel, and the blue point is the discretized distance point obtained by the RSSI ranging model. We fit the blue dot to get the space-time curve of each car. The slope of each space-time line is the average speed of the vehicle. The average speed of the vehicle is $13.13 \mathrm{~m} / \mathrm{s}$, and the relative error is $5 \%$. This method has good measurement accuracy. Fig. 9 is the continuous space-time graph obtained from discrete points. The average direct width between each space-time curve is calculated, and the average time headway is 4.16s. Therefore, the experimental calculation shows that the queue length of vehicles on the entrance road during the red light period is 6 vehicles. To sum up, this method can better measure the queue length during the intersection red light. 


\section{CONCLUSIONS}

Based on the ranging model based on the RSSI logarithmic distance attenuation model, we study the traffic state sensing method based on V2X wireless signals and validate the scheme's feasibility and accuracy through simulation. The main work of this paper is summarized as follows:

(1) By analyzing the wireless signal's outdoor propagation environment at the single-point intersection, combined with the transmission mode of the radio wave and the main factors affecting the attenuation, the wireless signal attenuation model is required for the experiment is determined as a logarithmic path attenuation model. An RSSI-based ranging model is obtained.

(2) The experiment is carried out by the simulation method. Firstly, the model of the intersection and its surrounding environment is established by the sumo simulation software. Then the OMNeT++ and veins are used to build the simulation platform of the vehicle network. In the simulation process, the original RSSI data of the imported road is collected first, and then the data is preprocessed by Gaussian filtering and culling abnormal data. The parameters of the ranging model are then calibrated. The relative error of the parameter is $3.75 \%$.

(3) Simulation experiment to measure the speed, traffic flow, traffic density of the westbound direct traffic at the common intersection, and the dedicated left-turn lane's queue length during the red light. The measurement results are close to the simulation setup's parameters, indicating the method feasibility and accuracy.

Our approach is done through simulation experiments, so there will certainly be some deviations from the actual case. We also take this issue into consideration. So we will explore this problem further in the next study. Our next step is to extend the simulation environment to practical application scenarios.

\section{Acknowledgement}

The author(s) received no specific help from somebody other than the listed authors for this study.

Funding Statement: This research was supported by the National Key Research and Development Program of China under Grant No. 2017YFB0102502, 2018YFB1600500 and 2017YFC0804803, the Beijing Municipal Natural Science Foundation under Grant No. L191001, the National Natural Science Foundation of China under Grant No. U20A20155 and 61822101, the Newton Advanced Fellow-ship under Grant No. 62061130221, the Young Elite Scientists Sponsorship Program by Hunan Provincial Department of Education under Grant No. $18 B 142$.

Conflicts of Interest: The authors declare that they have no conflicts of interest to report regarding the present study.

\section{References}

[1] G. Swathi, "A frame work for categorize the innumerable vulnerable nodes in mobile adhoc network," Computer Systems Science and Engineering, vol. 35, no.5, pp. 335-345, 2020. Article (CrossRef Link)

[2] C. Li, G. Wu, L. Xing, F. Zhu and L. Zhao, "An efficient certificateless aggregate signature scheme designed for Vanet," Computers, Materials \& Continua, vol. 63, no. 2, pp. 725-742, 2020. 
[3] Y. Mao, K. Li and D. Mao, "Application of wireless network positioning technology based on gps in geographic information measurement," Journal of New Media, vol. 2, no.3, pp. 131-135, 2020. Article (CrossRef Link)

[4] H. Liu, L. Qi and S. Rong, "Deterministic vessel automatic collision avoidance strategy evaluation modeling," Intelligent Automation \& Soft Computing, vol. 25, no.4, pp. 789-804, 2019.

[5] Y. Tang, W. Liu, Y. He, Y. Zhang and F. Zhang, "The development of generalized public bicycles in China and its role in the urban transportation system," Journal of Internet of Things, vol. 2, no. 3, pp. 101-107, 2020. Article (CrossRef Link)

[6] W. Hao, Y.J. Lin and Y. Cheng, "Signal Progression Model for Long Intersection Grouping and Coordination," IEEE Access, Vol. 6, pp. 30128-30136, 2018.Article (CrossRef Link)

[7] W. Hao, L. Liu, X.F. Yang, Y.F. Li, Y.J. Byon, "Reducing CACC Platoon Disturbances Caused by State Jitters by Combining Two Stages Driving State Recognition with Multiple Platoons Strategies and Risk Prediction,” IEEE Transactions on Intelligent Transportation Systems, pp. 1-11, 2020. Article (CrossRef Link)

[8] H. Zhao, Y.F. Li, W. Hao, Sr. Peeta, Y.B. Wang, "Evaluating the Effects of Switching Period of Communication Topologies and Delays on Electric Connected Vehicles Stream with Car-following Theory,” IEEE Transactions on Intelligent Transportation Systems, pp. 1-11, 2020. Article (CrossRef Link)

[9] C.X. Ma, W. Hao, A.B. Wang and H.X. Zhao, "Developing a Coordinated Signal Control System for Urban Ring Road Under the Vehicle-Infrastructure Connected Environment," IEEE Access, Vol. 6, pp. 52471-52478, 2018. Article (CrossRef Link)

[10] W. Hao, Z. Zhang, Z. Gao, K. Yi, J. Wang, "Research on mandatory lane-changing behavior in highway weaving sections," Journal of advanced transportation, Vol. 2020(6), pp. 1-9, 2020. Article (CrossRef Link)

[11] D. Kim and S. Kim, "Network-aided intelligent traffic steering in 5 g mobile networks," Computers, Materials \& Continua, vol. 65, no. 1, pp. 243-261, 2020. Article (CrossRef Link)

[12] S. Zhang, H. Luo, Z. Wu, Y. Sun, Y. Wang et al., "Efficient heavy hitters identification over speed traffic streams," Computers, Materials \& Continua, vol. 63, no. 1, pp. 213-222, 2020. Article (CrossRef Link)

[13] C. R. Tang, "Research and Analysis of WSN Node Location in Highway Traffic Based on Priority," Journal of Quantum Computing, vol. 2, no. 1, pp. 1-9, 2020. Article (CrossRef Link)

[14] P. Bahl, "RADAR: An In-Building RF-Based User Location and Tracking System," in Proc. of INFOCOM 2000. Nineteenth Annual Joint Conference of the IEEE Computer and Communications Societies, 2000. Article (CrossRef Link)

[15] J. Su, R. Xu, S. Yu, B. Wang, and J. Wang, "Idle slots skipped mechanism based tag identification algorithm with enhanced collision detection," KSII Transactions on Internet and Information Systems, vol. 14, no. 5, pp. 2294-2309, 2020. Article (CrossRef Link)

[16] J. Su, R. Xu, S. Yu, B. Wang, and J. Wang, "Redundant rule detection for software-defined networking," KSII Transactions on Internet and Information Systems, vol. 14, no. 6, pp. 2735-2751, 2020. Article (CrossRef Link)

[17] C. Ergen, T.H. Serhat and Kontik, "RSSI-Fingerprinting-Based Mobile Phone Localization with Route Constraints," IEEE Transactions on Vehicular Technology, Vol.63(1), pp. 423-428, 2014. Article (CrossRef Link)

[18] Y. Wang, J. Yang, H.B. Liu and Y.Y. Chen, "Measuring Human Queues Using Wi-Fi Signals," in Proc. of MobiCom: International Conference on Mobile Computing \& Networking, pp. 235-238, 2013. Article (CrossRef Link)

[19] W. Yan, Y.Y. Chen, and M. Richard, "Leveraging Wi-Fi Signals to Monitor Human Queues," IEEE Pervasive Computing, Vol.13(2), pp. 14-17, 2014. Article (CrossRef Link)

[20] Z. YANG, Z.M. ZHOU, Y.H. LIU, "From RSSI to CSI: Indoor Localization via Channel Response," ACM Computing Surveys, Vol.46(2), pp. 1-32, 2013. Article (CrossRef Link)

[21] H. Li, and M.X. Dong, "Learning Human Activities through Wi-Fi Channel State Information with Multiple Access Points," IEEE Communications Magazine, Vol.56(5), pp. 124-129, 2018. Article (CrossRef Link) 
[22] R. Zhou, and X. Lu, "Device-Free Presence Detection and Localization With SVM and CSI Fingerprinting," IEEE Sensors Journal, Vol.17(23), pp. 7990-7999, 2017. Article (CrossRef Link)

[23] X.Y. Wang, L.J. Gao and S.W. Mao, "CSI-Based Fingerprinting for Indoor Localization: A Deep Learning Approach," IEEE Transactions on Vehicular Technology, Vol.66(1), pp. 763-776, 2017. Article (CrossRef Link)

[24] Q.H. Gao, J. Wang and X.R. Ma, "CSI-Based Device-Free Wireless Localization and Activity Recognition Using Radio Image Features," IEEE Transactions on Vehicular Technology, Vol.66(11), pp. 10346-10356, 2017. Article (CrossRef Link)

[25] X.L. Zheng and J.L. Wang, "Design and Implementation of a CSI-Based Ubiquitous Smoking Detection System," IEEE-ACM TRANSACTIONS ON NETWORKING, Vol.25(6), pp. 3781-3793, 2017. Article (CrossRef Link)

[26] J.C. Wang, Z.S. Tian and X.L. Yang, "CSI Component Reconstruction-Based AoA Estimation for Subtle Human-Induced Reflection Under the TTW Scenario," IEEE Communications Letters, Vol.23(8), pp. 1393-1396, 2019. Article (CrossRef Link)

[27] Z.H. Chen and L. Zhang, "Wi-Fi CSI Based Passive Human Activity Recognition Using Attention Based BLSTM," IEEE Transactions on Mobile Computing, Vol.18(11), pp. 2714-2724, 2019. Article (CrossRef Link)

[28] S. Guha, K. Plarre, D. Lissner, S. Mitra, B. Krishna, P. Dutta, S. Kumar, "Auto Witness: Locating and tracking stolen property while tolerating GPS and radio outages," ACM Transactions on Sensor Networks, vol. 8(4), pp. 1-28, 2012. Article (CrossRef Link)

[29] X.H. Tian, S.J. Zhu, and S.J. Xiong, "Performance Analysis of Wi-Fi Indoor Localization with Channel State Information," IEEE Transactions on Mobile Computing, Vol.18(8), pp. 1870-1884, 2019. Article (CrossRef Link)

[30] Y.S.MA et al., "Wi-Fi Sensing with Channel State Information: A Survey," ACM Computing Surveys, Vol.52(3), pp. 1-36, 2019. Article (CrossRef Link)

[31] Mohanty, " VEPSD: A novel velocity estimation algorithm for next-generation wireless systems," IEEE Transactions on Wireless Communications, Vol.4(6), pp. 2655-2660, 2005.

Article (CrossRef Link)

[32] M. Waqas, S. Tu, S. U. Rehman, Z. Halim, S. Anwar et al., "Authentication of vehicles and road side units in intelligent transportation system," Computers, Materials \& Continua, vol. 64, no. 1, pp. 359-371, 2020.Article (CrossRef Link)

[33] J. Su, Z. G. Sheng, A. X. Liu and Y. R. Chen, "A group-based binary splitting algorithm for UHF RFID anti-collision systems," IEEE Transactions on Communications, vol. 68, no. 2, pp. 998-1012, 2020. Article (CrossRef Link)

[34] J. Su, Z. Sheng, A. X. Liu, and Y. Chen, "A partitioning approach to RFID identification," IEEE/ACM Transactions on Networking, vol. 28, no. 5, pp. 2160-2173, 2020. Article (CrossRef Link)

[35] J. Su, Z. Sheng, A. X. Liu, Y. Han, and Y. Chen, "Capture-aware identification of mobile RFID tags with unreliable channels," IEEE Transactions on Mobile Computing, pp. 1-1, 2020. Article (CrossRef Link)

[36] I. Constandache, R.R. Choudhury and I. Rhee, "Towards Mobile Phone Localization without WarDriving," in Proc. of INFOCOM, 2010 Article (CrossRef Link)

[37] A. Abdi, "On the estimation of the $\mathrm{K}$ parameter for the Rice fading distribution," IEEE Communications Letters, Vol.5(3), pp. 92-94, 2001. Article (CrossRef Link)

[38] J. Liu, X. Kang, C. Dong and F. Zhang, "Simulation of real-time path planning for large-scale transportation network using parallel computation," Intelligent Automation \& Soft Computing, vol. 25, no.1, pp. 65-77, 2019. Article (CrossRef Link)

[39] J.Sanguesa, "SensingTraffic Density Combining V2V and V2I Wireless Communications," Sensors, Vol.15(12), pp. 31794-31810, 2015. Article (CrossRef Link)

[40] H.Q. Liu, "A New Approach for Real-Time Traffic Delay Estimation Based on Cooperative Vehicle-Infrastructure Systems at the Signal Intersection," Arabian Journal for Science \& Engineering, Vol.44(3), pp. 2613-2625, 2019. Article (CrossRef Link) 
[41] W.Xu et al., "Online Method to Impute Missing Loop Detector Data for Urban Freeway Traffic Control," TRANSPORTATION RESEARCH RECORD, Vol.2593(2593), pp. 37-46, 2016. Article (CrossRef Link)

[42] K.Y.Jin et al., "A Single Node Vehicle Detection System Using an Adaptive Signal Adjustment Technique," in Proc. of 2017 20TH INTERNATIONAL SYMPOSIUM ON WIRELESS PERSONAL MULTIMEDIA COMMUNICATIONS (WPMC), pp. 349-353, 2017. Article (CrossRef Link)

[43] S. Chao, "Road Traffic Operation Assessment Based on Multi-source Floating Car Data Fusion," Journal of Tongji University, Vol.46(1), pp. 46-52, 2017. Article (CrossRef Link)

[44] Z. Gao et al., "Synergizing Appearance and Motion With Low Rank Representation for Vehicle Counting and Traffic Flow Analysis," IEEE Transactions on Intelligent Transportation Systems, Vol.19(8), pp. 2675-2685, 2018. Article (CrossRef Link)

[45] H. Chen, "Video Detection System of Traffic Flow toward Urban Roads," in Proc. of CSAI '18 Proceedings of the 2018 2nd International Conference on Computer Science and Artificial Intelligence, 2018

[46] H.P. Yin et al., "A Vision-Based Traffic Flow Detection Approach," in Proc. of PROCEEDINGS OF THE 2015 CHINESE INTELLIGENT SYSTEMS CONFERENCE, Vol. 359, pp. 143-152, 2015. Article (CrossRef Link)

[47] S.Li et al., "Vehicle counting and traffic flow parameter estimation for dense traffic scenes," IET Intelligent Transport Systems, Vol.14(12), pp. 1517-1523, 2020. Article (CrossRef Link)

[48] H.Q.Liu et al., "Data Feature Analysis of Non-Scanning Multi Target Millimeter-Wave Radar in Traffic Flow Detection Applications," SENSORS, Vol.18(9), pp. 2756, 2018. Article (CrossRef Link)

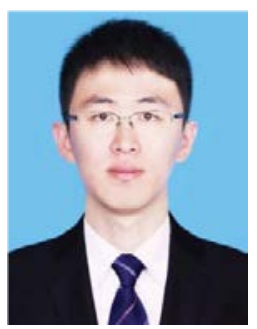

Xuting Duan (Member, IEEE) is currently a Lecturer with the School of Transportation Science and Engineering, Beihang University, Beijing, China. His current research interests include connected vehicles, vehicular ad-hoc networks, and vehicular localization.

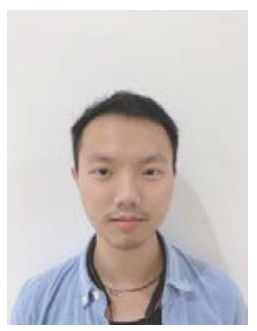

Hang Jiang received the B.S.degree with the School of Transportation Science and Engineering, Beihang University, Beijing, China. He is now a master student in the School of Transportation Science and Engineering, Beihang University, China. His research interests are 3D object detection and automatic driving environment perception.

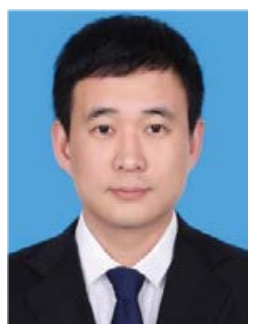

Daxin Tian (Senior Member, IEEE) is currently a Professor with the School of Transportation Science and Engineering, Beihang University, Beijing, China. His current research interests include mobile computing, intelligent transportation systems, vehicular adhoc networks, and swarm intelligence. 


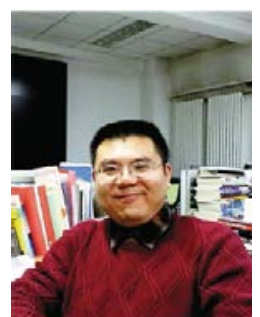

Jianshan Zhou received his B.Sc., M.Sc. and Ph.D. degrees in traffic information engineering and control from Beihang University, Beijing, China, in 2013, 2016 and 2020, respectively. From 2017 to 2018, he was a visiting research fellow with the school of informatics and engineering, University of Sussex, United Kingdom. In 2018, he was awarded the outstanding top-ten Ph.D. candidate prize from Beihang University. He also received the national scholarship in 2017 and 2019. He is currently a postdoctoral fellow supported by the "Zhuoyue" Program of Beihang University. He is the author or coauthor of more than 20 international scientific publications. His research interests include the modeling and optimization of vehicular communication networks and air-ground cooperative networks, the analysis and control of connected autonomous vehicles and intelligent transportation systems.

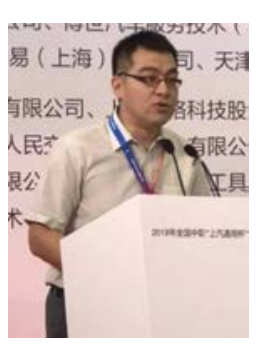

Gang Zhou is currently a senior engineer with Research Institute of Hightway Ministry of Transport, Beijing, China. His current research interests include intelligent transportation systems, and smart roads.
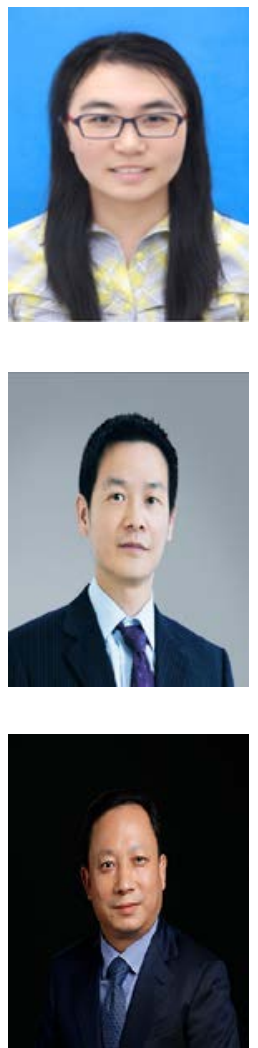

Wenjuan E is currently an accociate professor with Soochow University, Suzhou, China. Her current research interests include intelligent transportation systems, and cooperative vehicle infrastructure systems.
Yafu Sun is currently a vice president with China Transinfo Technology Co., Ltd, Beijing, China. His current research interests include intelligent transportation systems, and cooperative vehicle infrastructure systems.

Shudong Xia is currently the president with China Transinfo Technology Co., Ltd, Beijing, China. His current research interests include intelligent transportation systems, and connected vehicles. 